\title{
Non-Obvious, Post-Traumatic, Life-Threatening Bleeding in Two Elderly Patients
}

\author{
Rafael García Carretero* \\ Internal Medicine Department, Mostoles University Hospital, Spain
}

\begin{abstract}
The main complication of anticoagulant therapy is major bleeding. Clinicians are usually aware of these side effects and are careful when managing the therapeutic range of vitamin $\mathrm{K}$ antagonist drugs. But major bleeding, while lifethreatening, can be overlooked if there are no visible signs of bleeding. Two cases are described in which inaccurate diagnoses lead to inadequate treatment.
\end{abstract}

Keywords: Vitamin K antagonists, major bleeding, prothrombin complex concentrate

Received: 22 February 2017 / Accepted: 19 April 2017

\section{INTRODUCTION}

Vitamin K antagonists are widely used drugs. However, they have a narrow therapeutic range. Below the lower limit, the patient is not anticoagulated; above the upper limit, there is an increased risk of major bleeding. Diagnosing bleeding can be challenging, especially when haemorrhage is not evident. In patients who have been overdosed with anti-coagulants, serious consequences may arise, making an early and accurate diagnosis essential.

In patients needing a rapid reversal of these drugs, the effects of vitamin $\mathrm{K}$ antagonists can be reversed with oral vitamin $\mathrm{K}$ or prothrombin complex concentrate, depending on the severity of the case.

\section{CASE DESCRIPTION}

\section{Case 1}

A 75-year-old man, giving a five-month history of dizziness, light-headedness, muscular weakness, blurred vision and feeling faint. He was admitted to hospital due to a sudden loss of consciousness. He reported no prodromal symptoms and the relatives who were present when he fainted, said that the syncope was of a short duration. Shortly afterwards, he recovered spontaneously with no neurological symptoms. The patient had fallen, hitting his head and chest. Since he had atrial fibrillation and was taking the vitamin $\mathrm{K}$ antagonist, acenocoumarol, his relatives were concerned about the risk of bleeding after the fall. The patient had a history of Type 2 diabetes, right internal carotid artery stenosis. He suffered from chronic kidney disease, reported as Stage 3B, with a glomerular filtration rate of $40 \mathrm{ml} /$ $\mathrm{min} / 1.73 \mathrm{~m}^{2}$. He was taking aspirin $100 \mathrm{mg}$ daily, losar$\tan 100 \mathrm{mg}$ daily, atorvastatin $20 \mathrm{mg}$ daily, amlodipine $10 \mathrm{mg}$ daily, insulin glargine 16 IU daily and acenocoumarol $9 \mathrm{mg}$ weekly, taken daily at the same time of day, but adjusted every three weeks according to response.

On physical examination, he had a mild bleeding head injury and bruises on the left side of the face. His blood pressure was 139/51, heart rate was $65 \mathrm{bpm}$ and oxygen saturation was $95 \%$ in room air. On auscultation, heart sounds were arrhythmical, with no murmurs. Breath sounds were diminished on the left side, with hypoventilation in the basal and middle fields. Otherwise, the examination was normal.

On admission, routine blood tests showed a white blood cell count of 10,100/ $\mathrm{ll}$, a haemoglobin level of $12.7 \mathrm{~g} / \mathrm{dl}$ and a platelet count of $132,000 / \mu \mathrm{l}$. The International Normalized Ratio (INR) was 5.1. The remaining blood tests were normal. A chest X-ray revealed left-sided pleural effusion (Figure 1), with no rib fracture, and CT scan showed no subdural or epidural haematomas.

His doctor decided a thoracentesis should be performed, but since the pleural effusion was not causing dyspnoea or discomfort, it was considered appropriate 


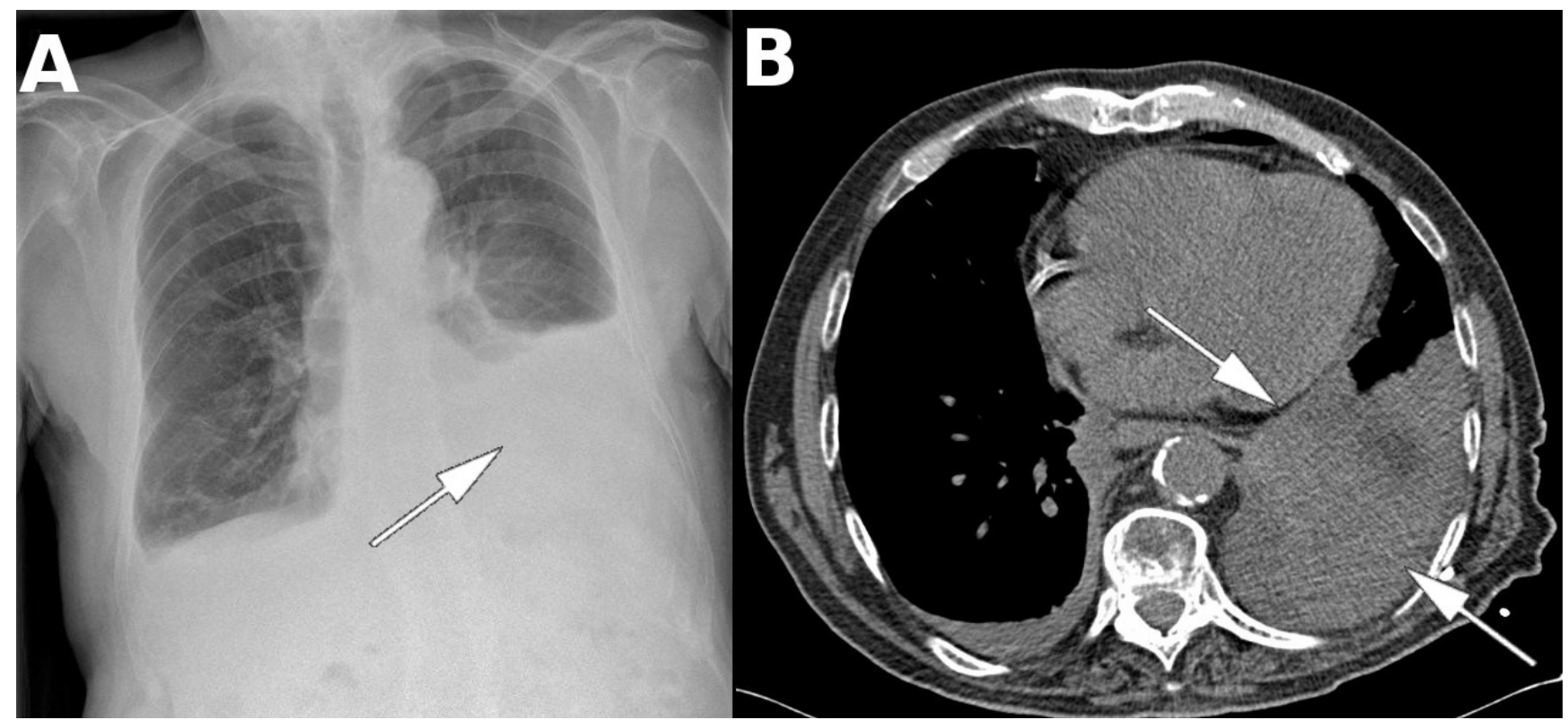

Fig. 1. A: The chest X-ray shows the left pleural effusion. B: The chest CT scan (without intravenous contrast) shows the left pleural effusion (arrows), the left lower lobe collapse and a small right pleural effusion.

to postpone this until the INR was normal. Acenocoumarol was withdrawn, and the patient was hospitalised.

Twenty-four hours after admission, further blood tests were performed, which revealed a haemoglobin level of $10.4 \mathrm{~g} / \mathrm{dl}$. It was noticed that the haemoglobin level had been $13.5 \mathrm{~g} / \mathrm{dl}$ a week earlier suggesting there was haemorrhagic pleural effusion. Prothrombin complex concentrate was immediately prescribed to reverse the effects of acenocoumarol. When the INR was normal, a diagnostic thoracentesis revealed a haemothorax, and the decision was taken to drain this through a pleural tube. A total amount of $1,800 \mathrm{ccs}$ of fluid were drained.

Over the following days, the haemothorax resolved. When the patient was discharged, he was referred to the Vascular Surgery Department (Alcorcon University Hospital, Madrid, Spain) for assessment of his previously known carotid stenosis, which was considered to be the most likely cause of the syncope.

\section{Case 2}

An 89-year-old man was admitted to hospital presenting with pain, swelling, redness and warmness in his left thigh. Two weeks before admission, he had tripped and fallen due to a defect in the pavement, hitting his left thigh. The patient had a medical history of atrial fibrillation and was taking acenocoumarol, $12 \mathrm{mg}$ weekly, taken daily at the same hour, but adjusted every three weeks, and amlodipine, $5 \mathrm{mg}$ daily.

His blood pressure was $155 / 93 \mathrm{mmHg}$, and heart rate was $58 \mathrm{bpm}$. His left thigh was enlarged compared to the right one, was warmer and appeared red and swollen. Otherwise, the examination was normal.

Initial laboratory tests showed a white blood cell count of 6,840/ $\mu \mathrm{l}$, a haemoglobin level of $7.1 \mathrm{~g} / \mathrm{dl}$ and a platelet count of $162,000 / \mu l$. The INR was 4.9. The remainder of the routine blood tests, including liver and kidney panels, and as C-reactive protein were normal. His doctor diagnosed cellulitis and prescribed amoxicillin/clavulanate $1 \mathrm{~g}$ every 8 hours. The patient was then admitted to hospital.

In the hospital, the case was taken over another doctor. A haematoma on the thigh was suspected and considered to be the cause of the severe anaemia. Prothrombin complex concentrate and two packed red blood cells transfusion were immediately prescribed. The patient underwent a CT scan (Figure 2), which showed a ruptured popliteal artery aneurysm, resulting in a nine $\mathrm{cm}$ haematoma on the left thigh.

The patient was referred to the Vascular Surgery Department (Alcorcon University Hospital, Madrid, Spain) where a metallic stent graft was inserted through the arteries of the left leg and deployed across an aneurysm (Figure 3). The patient was discharged four days later.

\section{DISCUSSION}

Vitamin K antagonists, such as warfarin and acenocoumarol, are widely used drugs for long-term anticoagulation therapy $[1,2]$. The use of these drugs should be 


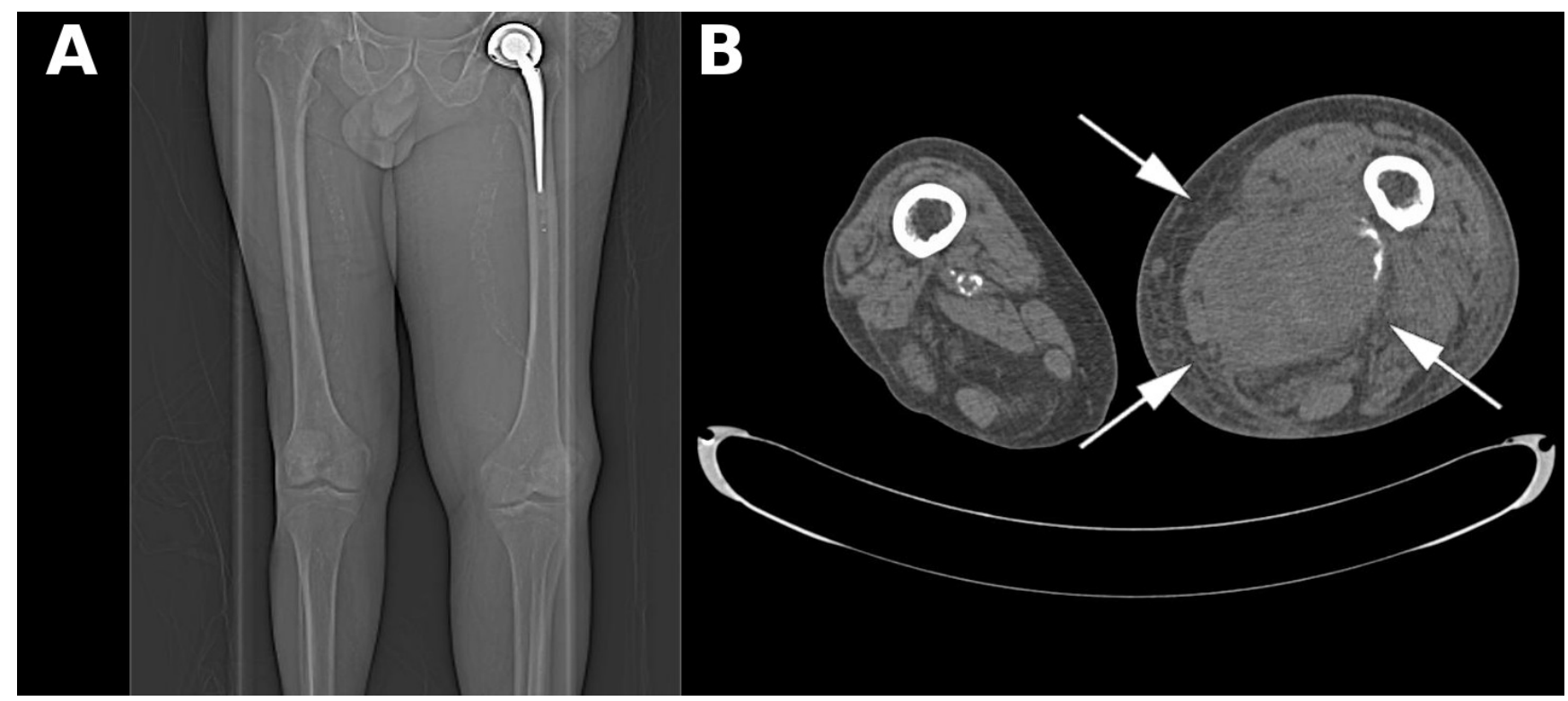

Fig. 2. A: CT scout view of both lower limbs, showing the enlargement of the left leg, compared to the right one. The increased size of the leg is even more apparent in the left thigh. B: CT of lower limbs (without intravenous contrast), showing the left haematoma (arrows). Note the atheromatous plaques in both popliteal arteries, and how the plaques draw an outline of the ruptured aneurysm.

undertaken with caution, given their narrow therapeutic range. The major side effect of vitamin $\mathrm{K}$ antagonist use is bleeding. The risk of bleeding is increased if the INR is out of normal range $[2,3]$. Clinicians should be aware of the subtle balance between bleeding complications and an insufficient anticoagulation status [4].

Major bleeding can be life-threatening [2], but bleeding is not always obvious. Bleeding wounds, skin bruises, gastrointestinal bleeding and brain haemorrhages can be easily noticed. However, retroperitoneal haemorrhages, post-traumatic haematomas and unnoticed rupture of an arterial aneurysm are a few examples of occult bleeding.

In both cases described, initial diagnoses were inaccurate. The first patient was admitted to hospital because of a syncope.

The main risk of a patient on acenocoumarol therapy is intracranial bleeding, which was why the patient underwent a brain CT scan. The patient was properly managed, but the clinician overlooked other conditions related to internal bleeding.

On the other hand, pleural effusion must be assessed if the aetiology is uncertain [5], and thoracentesis should not have been delayed. The only treatment was the withdrawal of acenocoumarol while waiting for the INR to drop to normal limits. The possibility of post-traumatic haemorrhagic pleural effusion in an anticoagulated patient was not considered. The patient was stabilised, but since the INR was out of the normal range, it would have been reasonable to believe that the haemorrhagic pleural effusion could worsen.

The diagnoses of head injury and pleural effusion of unknown origin were proposed. Unfortunately proper management, comprising the infusion of prothrombin complex concentrate before inserting a drainage intercostal tube, was delayed for twenty-four hours. Haemothorax is a risk factor for the development of complications such as empyema and fibrothorax, and the delay in proscribing the above must be viewed as a diagnostic error [6].

The second patient presented with severe anaemia, yet had a good tolerance. This tolerance may have been a confounding factor, despite the INR being out of the normal range. The initial diagnosis, that of cellulitis of the left thigh, was inaccurate. Peripheral pulses were weak but present and symmetrical. However, the clinical course of the anaemia had been progressive, and this was not linked to the trauma to the swollen leg. Therefore, two different diagnoses, cellulitis of the left thigh and chronic anaemia of unknown origin, were established.

On admission to hospital, the patient was put under the care of another doctor. The patient underwent a CT that confirmed the primary suspicion of post-traumatic internal bleeding. After two packed red blood cells and prothrombin complex concentrate had been ad- 


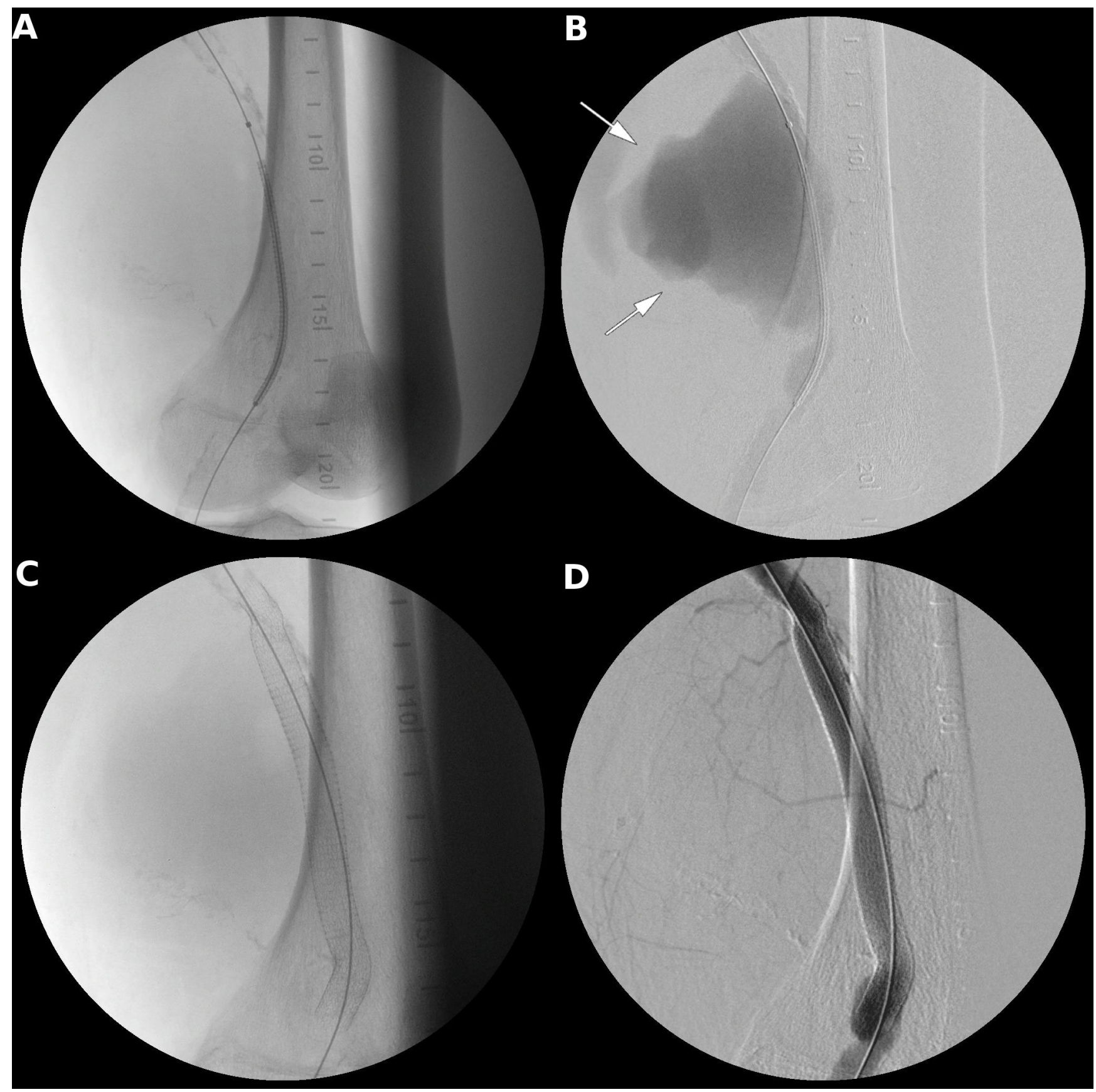

Fig. 3. A: Angiogram showing the catheterisation of the popliteal artery. B: Active extravasation of arterial contrast, outlining the haematoma. C: Covered stent placed to repair an aneurysm. D: The angiogram shows the lack of contrast leakage from the stent graft.

ministered, a vascular surgeon oversaw the treatment of the ruptured arterial aneurysm. (Figure 3).

\section{CONCLUSIONS}

Clinicians must be aware of the main side effects of vitamin $\mathrm{K}$ antagonists, especially when the INR is above the upper normal limit.
Some internal bleeding can go unnoticed. Students, interns and residents must be taught to link symptoms, signs and tests to establish a proper, accurate diagnose when managing the risk of bleeding in an anticoagulated patient.

Major bleeding in an anticoagulated patient can be life-threatening or leave serious sequelae. It is, therefore, critical to diagnose the origin of the bleeding. 
Available online at: www.jccm.ro

Anaemia, empyema, fibrothorax and infected haematomas are some of the possible complications of unobserved bleeding.

We believe that these two cases have a noteworthy educational value as they highlight the relationship between vitamin $\mathrm{K}$ antagonists and the risk of undetected bleeding.

\section{CONFLICT OF INTEREST}

The author has not any competing interests in the manuscript.

\section{REFERENCES}

1. Camm AJ, Lip GYH, De Caterina R, et al. 2012 focused update of the ESC Guidelines for the management of atrial fibrillation: an update of the 2010 ESC Guidelines for the management of atrial fibrillation. Developed with the special contribution of the European Heart Rhythm Association. Eur Heart J. 2012;33:2719
The Journal of Critical Care Medicine 2017;3(2) • 83

-47. doi: 10.1093/eurheartj/ehs253

2. Holbrook A, Schulman S, Witt DM, et al. Evidence-based management of anticoagulant therapy: Antithrombotic therapy and prevention of thrombosis, 9th ed: American College of Chest Physicians Evidence-Based Clinical Practice Guidelines. Chest. 2012;141:e152S-e184S. doi: 10.1378/chest.11-2295.

3. Garcia D, Crowther MA, Ageno W. Practical management of coagulopathy associated with warfarin. BMJ. 2010;340:c1813. doi: 10.1136/bmj.c1813.

4. UferM. Comparative Pharmacokinetics of Vitamin KAntagonists: warfarin, phenprocoumon and acenocoumarol. Clin Pharmacokinet. 2005;44:1227-46. doi: 10.2165/00003088200544120-00003

5. Villena Garrido V, Cases Viedma E, Fernandez Villar A, et al. Recommendations of diagnosis and treatment of pleural effusion. Update. Arch Bronconeumol. 2014;50:235-49. doi: 10.1016/j.arbres.2014.01.016.

6. Huggins JT, Sahn SA. Causes and management of pleural fibrosis. Respirology. 2004;9:441-7. doi: 10.1111/j.14401843.2004.00630.x 